\title{
EINSTEIN-WEYL STRUCTURES ON ALMOST CONTACT METRIC MANIFOLDS
}

Dedicated to Professor Shûkichi Tanno on his sixtieth birthday

By

Fumio NARITA

\section{Introduction}

Pedersen and Swann [7] and Higa [2] studied the existence of an EinsteinWeyl structure on principal circle bundles over Einstein Kaehler manifolds of positive scalar curvature and obtained many examples of Einstein-Weyl spaces. In a Riemannian submersion $\pi: M \rightarrow N$ with totally geodesic fibers of dimension one over an Einstein manifold $N$, we studied the relation between an EinsteinWeyl structure and a Sasakian structure of $M$ (cf. [5]).

On the other hand, in [4], we investigated some geometric structures of a Riemannian submersion $\pi: M \rightarrow N$, where $M$ is a CR-submanifold of a locally conformal Kaehler manifold $L$. Let $M$ be a leaf of the canonical foliation $\mathscr{M}$ given by the Lee form $\omega=0$ of a locally conformal Kaehler manifold $L$. Then $M$ admits an almost contact metric structure. We obtained a necessary and sufficient condition for the manifold $M$ to admits a Sasakian structure.

In this paper, we shall study the existence of an Einstein-Weyl structure on an almost contact metric manifold. Let $M$ be a complete and simply connected Sasakian manifold with constant $\phi$-sectional curvature $k$. We show that if $k \geq 1$, then $M$ admits an Einstein-Weyl structure.

Next, we assume that all local Kaehler metrics $g^{\prime}=e^{-r} g$ of a locally conformal Kaehler manifold $L$ have the same constant nonnegative holomorphic sectional curvature $\rho$. We show that if the induced almost contact metric structure of a leaf $M$ of the canonical foliation $\mathscr{M}$ of $L$ is Sasakian, then $M$ admits an Einstein-Weyl structure. Finally, we discuss the existence of an Einstein-Weyl structure on a leaf of the canonical foliation of a complete and simply connected generalized Hopf manifold.

Received June 5, 1996

Revised September 27, 1996 


\section{Preliminaries}

Firstly, we give the definition of an Einstein-Weyl space. Let $(M, g)$ be a Riemannian manifold. Let $D$ be a torsion-free affine connection on $M$. A manifold $M$ is said to have an Einstein-Weyl structure if there exist a 1 -form $\mu$ and a function $\Lambda$ on $M$ such that

$$
D g=\mu \otimes g \text { and } \quad{ }^{D} \operatorname{Ric}(X, Y)+{ }^{D} \operatorname{Ric}(Y, X)=\Lambda g(X, Y),
$$

where ${ }^{D}$ Ric is the Ricci tensor of $D$. Since $D$ is not a metric connection, the Ricci tensor is not necessarily symmetric. The Einstein-Weyl equation is conformally invariant. Let $\nabla$ be the Levi-Civita connection of $g$. We define a vector field $E$ by $g(X, E)=\mu(X)$. Then, since $D g=\mu \otimes g$, we have

$$
D_{X} Y=\nabla_{X} Y-\frac{1}{2} \mu(X) Y-\frac{1}{2} \mu(Y) X+\frac{1}{2} g(X, Y) E .
$$

Let ${ }^{D} R$ and $R$ be the curvature tensor of $D$ and $\nabla$ respectively. Then, we have

$$
\begin{aligned}
{ }^{D_{R}(X, Y) Z=} & R(X, Y) Z-\frac{1}{2}\left\{\left[\left(\nabla_{X} \mu\right) Z+\frac{1}{2} \mu(X) \mu(Z)\right] Y\right. \\
& -\left[\left(\nabla_{Y} \mu\right) Z+\frac{1}{2} \mu(Y) \mu(Z)\right] X+\left(\left(\nabla_{X} \mu\right) Y\right) Z-\left(\left(\nabla_{Y} \mu\right) X\right) Z \\
& \left.-g(Y, Z)\left(\nabla_{X} E+\frac{1}{2} \mu(X) E\right)+g(X, Z)\left(\nabla_{Y} E+\frac{1}{2} \mu(Y) E\right)\right\} \\
& -\frac{1}{4}|\mu|^{2}(g(Y, Z) X-g(X, Z) Y),
\end{aligned}
$$

where $X, Y$ and $Z$ are any vector fields on $M$.

Next, we give the definition of an almost contact metric manifold. A Riemannian manifold $(M, g)$ is said to be an almost contact metric manifold if there exist a tensor field $\phi$ of type $(1,1)$, a unit vector field $V$ and a 1 -form $\eta$ such that

$$
\begin{gathered}
\eta(V)=1, \quad \phi^{2} X=-X+\eta(X) V, \\
g(\phi X, \phi Y)=g(X, Y)-\eta(X) \eta(Y),
\end{gathered}
$$

for any vector fields $X, Y$ on $M$ (cf. [1]).

For an almost contact metric structure $(\phi, V, \eta, g)$ on $M$ we put $\Phi(X, Y)=$ $g(X, \phi Y)$. An almost contact metric structure is said to be:

Contact metric if $d \eta=\Phi$.

$K$-contact if $d \eta=\Phi$ and $V$ is a Killing vector field with respect to $g$. 
Sasakian if $d \eta=\Phi$ and $N_{\phi}+2 d \eta \otimes V=0$, where $N_{\phi}(X, Y)=[\phi X, \phi Y]-$ $\phi[\phi X, Y]-\phi[X, \phi Y]+\phi^{2}[X, Y]$.

If the Ricci tensor Ric of a contact metric manifold $M$ is of the form

$$
\operatorname{Ric}(X, Y)=\beta g(X, Y)+\gamma \eta(X) \eta(Y),
$$

$\beta$ and $\gamma$ being constant, then $M$ is called $\eta$-Einstein manifold.

Next, let $L$ be an almost Hermitian manifold with metric $g$, complex structure $J$ and the fundamental 2-form $\Omega$. The manifold $L$ is said to be a locally conformal Kaehler manifold if every $x \in L$ has an open neighborhood $U$ with a differentiable function $r: U \rightarrow R$ such that $g_{U}^{\prime}=\left.e^{-r} g\right|_{U}$ is a Kaehler metric on $U$. If we can take $U=L$, the manifold is globally conformal Kaehler. The locally conformal Kaehler manifold $L$ is characterized by

$$
N_{J}=0, \quad d \Omega=\omega \wedge \Omega, \quad d \omega=0,
$$

where $N_{J}$ is the Nijenhuis tensor of $J$ and $\omega$ is a globally defined 1-form on $L$. We call $\omega$ the Lee form. Since for $\operatorname{dim} L=2$ we have $d \Omega=0$, we may suppose $\operatorname{dim} L \geq 4$. Next we define a Lee vector field $B$ by

$$
g(X, B)=\omega(X) \text {. }
$$

The Weyl connection ${ }^{W} \nabla$ is the linear connection defined by

$$
{ }^{W} \nabla_{X} Y:=\nabla_{X} Y-\frac{1}{2} \omega(X) Y-\frac{1}{2} \omega(Y) X+\frac{1}{2} g(X, Y) B
$$

where $\nabla$ is the Levi-Civita connection of $g .{ }^{W} \nabla$ is the Levi-Civita connection of a local Kaehler metric $g^{\prime}$. It is shown in [9] that an almost Hermitian manifold $L$ is a locally conformal Kaehler if and only if there is a closed 1-form $\omega$ on $L$ such that

$$
{ }^{W} \nabla_{X} J=0 \text {. }
$$

Let $L$ be a locally conformal Kaehler manifold. Let $R^{L}$ be the curvature tensor field of the connection $\nabla$ and ${ }^{W} R$ the curvature tensor field of the Weyl connection ${ }^{W} \nabla$. Since $d \omega=0$, we obtain $\left(\nabla_{X} \omega\right) Y-\left(\nabla_{Y} \omega\right) X=0$. Thus, we have

$$
\begin{aligned}
(10){ }^{W} R(X, Y) Z= & R^{L}(X, Y) Z-\frac{1}{2}\left\{\left[\left(\nabla_{X} \omega\right) Z+\frac{1}{2} \omega(X) \omega(Z)\right] Y\right. \\
& -\left[\left(\nabla_{Y} \omega\right) Z+\frac{1}{2} \omega(Y) \omega(Z)\right] X-g(Y, Z)\left(\nabla_{X} B+\frac{1}{2} \omega(X) B\right) \\
& \left.+g(X, Z)\left(\nabla_{Y} B+\frac{1}{2} \omega(Y) B\right)\right\}-\frac{1}{4}|\omega|^{2}(g(Y, Z) X-g(X, Z) Y),
\end{aligned}
$$

where $X, Y$ and $Z$ are any vector fields on $L$ (cf. [11]). 
Let $\mathscr{M}$ be the foliation given by $\omega=0$ of a locally conformal Kaehler manifold $(L, J, g) . \mathscr{M}$ is called the canonical foliation.

A locally conformal Kaehler manifold $(L, J, g)$ is said to be a generalized Hopf manifold if the Lee form is parallel, that is $\nabla \omega=0(\omega \neq 0)$.

Let $M$ be a submanifold of a Riemannian manifold $L$. We denote by the same $g$ the Riemannian metric tensor field induced on $M$ from that of $L$. Let $\nabla^{M}$ denote covariant differentiation of $M$. Then the Gauss formula for $M$ is written as

$$
\nabla_{X} Y=\nabla_{X}^{M} Y+\sigma(X, Y)
$$

for any tangent vector fields $X, Y$ on $M$ where $\sigma$ denotes the second fundamental form of $M$ in $L$.

Let $R^{M}$ be the Riemannian curvature tensor field of $M$. Then we have the equation of Gauss

$R^{L}(W, Z, X, Y)=R^{M}(W, Z, X, Y)+g(\sigma(X, Z), \sigma(Y, W))-g(\sigma(Y, Z), \sigma(X, W))$.

\section{Einstein-Weyl structures}

Let $(M, \phi, V, \eta, g)$ be an almost contact metric manifold of dimension $2 n+1$. Let $\nabla^{M}$ be the Levi-Civita connection of $g$ and $R i c^{M}$ be the Ricci tensor of $\nabla^{M}$. Then we obtain the following result.

THEOREM 1. Let $(M, \phi, V, \eta, g)$ be an almost contact metric manifold satisfying $\nabla_{X}^{M} V=-\phi(X)$. If $\operatorname{Ric}^{M}(X, Y)=\beta g(X, Y)+\gamma \eta(X) \eta(Y)$, where $\beta$ and $\gamma$ are constant, and $\gamma \leq 0$, then $M$ admits an Einstein-Weyl structure.

Proof. We define 1 -form $\mu$ by $\mu=f \eta$, where $f$ is a function on $M$. Let $E$ be the dual vector field of $\mu$. We define the connection $D$ by

$$
D_{X} Y=\nabla_{X}^{M} Y-\frac{1}{2} \mu(X) Y-\frac{1}{2} \mu(Y) X+\frac{1}{2} g(X, Y) E .
$$

Then $D$ is a torsion-free connection and $D g=\mu \otimes g$. Let ${ }^{D}$ Ric be the Ricci tensor of $D$. From (3), we obtain

$$
\begin{aligned}
{ }^{D} \operatorname{Ric}(X, Y)= & \operatorname{Ric}^{M}(X, Y)+n\left(\nabla_{X}^{M} \mu\right) Y-\frac{1}{2}\left(\nabla_{Y}^{M} \mu\right) X \\
& +\frac{1}{4}(2 n-1) \mu(X) \mu(Y)+g(X, Y)\left(\frac{1}{2} \operatorname{div} E-\frac{1}{4}(2 n-1)|\mu|^{2}\right)(\mathrm{cf} .[7]) .
\end{aligned}
$$


Let $V, X_{1}, \phi X_{1}, \ldots, X_{n}, \phi X_{n}$ be an orthonormal basis of $T_{p} M$. Since $\nabla_{X}^{M} V=$ $-\phi(X)$, for $j=1, \ldots, n$, we get $\nabla_{V}^{M} V=0$ and $g\left(\nabla_{X_{j}}^{M} X_{j}, V\right)=-g\left(\nabla_{X_{j}}^{M} V, X_{j}\right)=0$. Thus we have $\left(\nabla_{X_{j}}^{M} \mu\right) V=X_{j}(f),\left(\nabla_{V}^{M} \mu\right)\left(X_{j}\right)=0,\left(\nabla_{V}^{M} \mu\right) V=V(f),\left(\nabla_{X_{i}}^{M} \mu\right) X_{j}=0$ for all $i, j,\left(\nabla_{X_{i}}^{M} \mu\right)\left(\phi X_{j}\right)=0$ for $i \neq j$ and $\left(\nabla_{X_{j}}^{M} \mu\right)\left(\phi X_{j}\right)+\left(\nabla_{\phi X_{j}}^{M} \mu\right) X_{j}=0$. From these, we have

$$
\begin{aligned}
& { }^{D} \operatorname{Ric}\left(X_{j}, V\right)+{ }^{D} \operatorname{Ric}\left(V, X_{j}\right)=\frac{1}{2}(2 n-1) X_{j}(f), \\
& { }^{D} \operatorname{Ric}\left(\phi X_{j}, V\right)+{ }^{D} \operatorname{Ric}\left(V, \phi X_{j}\right)=\frac{1}{2}(2 n-1) \phi X_{j}(f), \\
& 2 \cdot{ }^{D} \operatorname{Ric}(V, V)=2(\beta+\gamma)+\frac{1}{2}(2 n-1)\left(2 V(f)+f^{2}\right)+\operatorname{div} E-\frac{1}{2}(2 n-1) f^{2} \\
& 2 \cdot{ }^{D} \operatorname{Ric}\left(X_{j}, X_{j}\right)=2 \beta+\operatorname{div} E-\frac{1}{2}(2 n-1) f^{2},
\end{aligned}
$$

and

$$
2 \cdot{ }^{D} \operatorname{Ric}\left(\phi X_{j}, \phi X_{j}\right)=2 \beta+\operatorname{div} E-\frac{1}{2}(2 n-1) f^{2} .
$$

We set $f^{2}=-(4 /(2 n-1)) \gamma$. Then $V(f)=0, X_{j}(f)=\phi X_{j}(f)=0$ for $j=1, \ldots, n$ and $\operatorname{div} E=V \cdot \mu(V)=V(f)=0$. Thus, by using equations (15)-(19), we obtain

$$
{ }^{D} \operatorname{Ric}(X, Y)+{ }^{D} \operatorname{Ric}(Y, X)=\Lambda g(X, Y),
$$

where $X, Y$ are tangent vectors of $M$ and $\Lambda=2(\beta+\gamma)$. Therefore $M$ admits an Einstein-Weyl structure.

REMARK 1. $K$-contact manifold and Sasakian manifold satisfy the condition $\nabla_{X}^{M} V=-\phi(X)$ (cf. [1]). Let $\pi: M^{2 n+1} \rightarrow N^{2 n}$ be a Riemannian submersion with totally geodesic fibers of dimension one over an Einstein manifold $N^{2 n}$ such that $\operatorname{Ric}^{N}(\tilde{X}, \tilde{Y})=c \tilde{g}(\tilde{X}, \tilde{Y})$. Moreover, we assume that $\left(M^{2 n+1}, \phi, V, \eta, g\right)$ is a standard Sasakian manifold. Let $V, X_{1}, \phi X_{1}, \ldots, X_{n}, \phi X_{n}$ be an orthonormal basis of $T_{p} M$. Then we get $\operatorname{Ric}^{M}\left(X_{j}, X_{j}\right)=\operatorname{Ric}^{M}\left(\phi X_{j}, \phi X_{j}\right)=c-2, \operatorname{Ric}^{M}(V, V)=2 n$, $\operatorname{Ric}^{M}\left(X_{j}, V\right)=\operatorname{Ric}^{M}\left(\phi X_{j}, V\right)=0, \operatorname{Ric}^{M}\left(X_{i}, \phi X_{j}\right)=0$ for all $i, j$ and $\operatorname{Ric}^{M}\left(X_{i}, X_{j}\right)=$ $\operatorname{Ric}^{M}\left(\phi X_{i}, \phi X_{j}\right)=0$ for $i \neq j$. (cf. [5]). Therefore $M$ is an $\eta$-Einstein manifold such that $\operatorname{Ric}^{M}(X, Y)=(c-2) g(X, Y)+(2 n-c+2) \eta(X) \eta(Y)$. If the scalar curvature $\tilde{s}$ of $N$ is $\tilde{s} \geq 4 n(n+1)$, then $2 n-c+2=(1 /(2 n))\left(4 n^{2}+4 n-\tilde{s}\right) \leq 0$. Thus, as a corollary of Theorem 1, we obtain Theorem 2 in [5].

Next, let $(M, \phi, V, \eta, g)$ be a Sasakian manifold of constant $\phi$-sectional curvature $k$ and $\operatorname{dim} M=2 n+1$. The curvature of $M$ is 


$$
\begin{aligned}
R^{M}(X, Y, Z, W)= & \frac{k+3}{4}\{g(X, Z) g(Y, W)-g(X, W) g(Y, Z)\} \\
& -\frac{k-1}{4}\{\eta(X) \eta(Z) g(Y, W)-\eta(Y) \eta(Z) g(X, W) \\
& +g(X, Z) \eta(Y) g(V, W)-g(Y, Z) \eta(X) g(V, W) \\
& +g(\phi Y, Z) g(\phi X, W)-g(\phi X, Z) g(\phi Y, W) \\
& +2 g(X, \phi Y) g(\phi Z, W)\} \quad \text { (cf. [12] }) .
\end{aligned}
$$

From this equation, we get

$$
\operatorname{Ric}^{M}(X, Y)=\beta g(X, Y)+\gamma \eta(X) \eta(Y),
$$

where $\beta=((n+1) / 2) k+((3 n-1) / 2)$ and $\gamma=-((n+1) / 2)(k-1)$.

Let $S^{2 n+1}$ be the unit sphere in $C^{n+1}$ and $\tau$ be real number such that $\tau>0$. Let $(J, h)$ be the flat Kaehler structure on $C^{n+1}$ and $U$ be the unit normal vector field of $S^{2 n+1}$ in $C^{n+1}$. We define tensor field $\phi$ and 1 -form $\bar{\eta}$ on $S^{2 n+1}$ by $J(X)=$ $\phi(X)-\bar{\eta}(X) U$. And we put $V=(1 / \tau) J U, \eta=\tau \bar{\eta}$ and $g=\tau h^{\prime}+\tau(\tau-1) \bar{\eta} \otimes \bar{\eta}$, where $h^{\prime}$ is the induced metric on $S^{2 n+1}$ by $h$. Then $(\phi, V, \eta, g)$ is a Sasakian structure with constant $\phi$-sectional curvature $k=(4 / \tau)-3$ and we denote $S^{2 n+1}$ with this structure by $S^{2 n+1}(k)$ (cf. [8]). Thus we have (22)] and $\nabla_{X}^{S} V=-\phi(X)$, where $\nabla^{S}$ is the Levi-Civita connection of $g$. From a Theorem of Tanno [8] and Theorem 1, we obtain the following result.

THEOREM 2. Let $M$ be a complete and simply connected Sasakian manifold with constant $\phi$-sectional curvature $k$. If $k \geq 1$, then $M$ admits an Einstein-Weyl structure.

REMARK 2. Let $(\phi, V, \eta, g)$ be a Sasakian structure of the sphere $\left(S^{2 n+1}(k), g\right)$. In the Hopf fibration $\pi:\left(S^{2 n+1}(k), g\right) \rightarrow\left(P_{n}(C)(k+3), \tilde{g}\right)$, for constant $a \neq 0$, we define a Riemannian metric $g_{a}$ by $g_{a}=\pi^{*} \tilde{g}+a^{2} \eta \otimes \eta$ (cf. [2]). We set $\tilde{V}=(1 / a) V$ and $\tilde{\eta}=a \eta$. Then $d \tilde{\eta}=a \tilde{\Phi}$, where $\tilde{\Phi}(X, Y)=g_{a}(X, \phi Y)$. Let $A$ be the integrability tensor of the Riemannian submersion $\pi_{a}:\left(S^{2 n+1}(k), g_{a}\right) \rightarrow$ $\left(P_{n}(C)(k+3), \tilde{g}\right)$ with totally geodesic fibers. Let $R^{S}$ and $R^{P}$ denote the curvature tensors of $\left(S^{2 n+1}(k), g_{a}\right)$ and $\left(P_{n}(C)(k+3), \tilde{g}\right)$ respectively. We recall the following curvature identity.

$$
\begin{aligned}
R^{S}(W, Z, X, Y)= & R^{P}(\tilde{W}, \tilde{Z}, \tilde{X}, \tilde{Y})-g_{a}\left(A_{Y} Z, A_{X} W\right) \\
& +g_{a}\left(A_{X} Z, A_{Y} W\right)+2 g_{a}\left(A_{X} Y, A_{Z} W\right)
\end{aligned}
$$


where $\tilde{X}, \tilde{Y}, \tilde{Z}$ and $\tilde{W}$ are tangent vector fields on $P_{n}(C)(k+3)$ and $X, Y, Z$ and $W$ are the horizontal lifts of $\tilde{X}, \tilde{Y}, \tilde{Z}$ and $\tilde{W}$ respectively (cf. [6]). For a function $f$ on $S^{2 n+1}(k)$, we put $\mu=f \eta$. We define the connection $\bar{D}$, by

$$
\bar{D}_{X} Y=\nabla_{X}^{a} Y-\frac{1}{2} \mu(X) Y-\frac{1}{2} \mu(Y) X+\frac{1}{2} g_{a}(X, Y) E,
$$

where $\nabla^{a}$ is the Levi-Civita connection of $g_{a}$ and $E$ is the dual vector field of $\mu$. Let ${ }^{\bar{D}} R i c^{a}$ be the Ricci tensor of $\bar{D}$. Since $A_{X} Y=(1 / 2) \mathscr{V}[X, Y]$ (cf. [6]) and $\tilde{V}$ is a unit vertical vector field, we get $A_{X} Y=-d \tilde{\eta}(X, Y) \tilde{V}=-a g_{a}(X, \phi Y) \tilde{V}$, where $X, Y$ are horizontal vector fields. Using $|\mu|^{2}=\left(f^{2} / a^{2}\right)$, for an orthonormal basis $\tilde{V}, X_{1}, \phi X_{1}, \ldots, X_{n}, \phi X_{n}$ of $T_{p} S^{2 n+1}(k)$, we get following.

$$
\begin{gathered}
2 \cdot \bar{D}_{R i c^{a}}(\tilde{V}, \tilde{V})=4 n a^{2}+\frac{1}{2}(2 n-1)\left(\frac{2}{a} \tilde{V}(f)+\frac{f^{2}}{a^{2}}\right)+\operatorname{div} E-\frac{1}{2}(2 n-1) \frac{f^{2}}{a^{2}}, \\
2 \cdot{ }^{\bar{D}_{R i c}}{ }^{a}\left(X_{j}, X_{j}\right)=(n+1)(k+3)-4 a^{2}+\operatorname{div} E-\frac{1}{2}(2 n-1) \frac{f^{2}}{a^{2}}
\end{gathered}
$$

and

$$
{ }^{\bar{D}} \operatorname{Ric}^{a}\left(X_{j}, \tilde{V}\right)+{ }^{\bar{D}} R i c^{a}\left(\tilde{V}, X_{j}\right)=\frac{1}{2}(2 n-1) X_{j}\left(\frac{f}{a}\right) .
$$

For $\phi X_{j}$, we also have same equations.

Let constant $a$ be $(k+3) / 4 \geq a^{2}$ and set $f^{2}=\left(2(n+1) a^{2} /(2 n-1)\right) \times$ $\left(k+3-4 a^{2}\right)$. Then we have

$$
{ }^{\bar{D}} \operatorname{Ric}^{a}(X, Y)+{ }^{\bar{D}} \operatorname{Ric}^{a}(Y, X)=\Lambda g_{a}(X, Y),
$$

where $\Lambda=4 n a^{2}$. Therefore, for $k>-3,\left(S^{2 n+1}(k), g_{a}, \mu\right)$ admits an Einstein-Weyl structure but not Sasakian for $a \neq 1$.

\section{Foliations of locally conformal Kaehler manifolds}

As an application of Theorem 1 and Theorem 2, we consider the canonical foliation of a locally conformal Kaehler manifold. Let $(L, J, g)$ be a locally conformal Kaehler manifold of dimension $2 n+2$ and $\omega$ the Lee form and $\mathscr{M}$ the canonical foliation given by $\omega=0$. Let $M$ be a leaf of the canonical foliation $\mathscr{M}$, that is $M$ is an orientable real hypersurface of $L$. Let $B$ be the Lee vector field. We set $C=(B /|\omega|), V=J C, \eta(X)=g(X, V)$ and $J X=\phi X-\eta(X) C$. It is known that every orientable real hypersurface of an almost Hermitian manifold has an almost contact metric structure $(\phi, V, \eta, g)$ (cf. [1], [10]). 
We show the following theorem.

THEOREM 3. Let $(L, g)$ be a locally conformal Kaehler manifold and assume that all its local Kaehler metrics $g^{\prime}=e^{-r} g$ have the same constant nonnegative holomorphic sectional curvature $\rho$. Let $M$ be a leaf of the canonical foliation $\mathscr{M}$. If the induced almost contact metric structure of $M$ is Sasakian, then $M$ admits an Einstein-Weyl structure.

ProOF. It is known that $M$ admits a Sasakian structure if and only if

$$
\sigma(X, Y)=-\left(\left(\frac{1}{2}|\omega|-1\right) g(X, Y)+\alpha \eta(X) \eta(Y)\right) C
$$

where $\alpha$ is a function on $M$ (cf. [4]). Let $C, V, X_{1}, J X_{1}, \ldots, X_{n}, J X_{n}$ be an orthonormal basis of $T_{p} L$ such that $V, X_{1}, \phi X_{1}, \ldots, X_{n}, \phi X_{n}\left(\phi X_{i}=J X_{i}\right)$ form an orthonormal basis of $T_{p} M$. We denote the Ricci tensor of $\nabla$ of $L$ by $R i c^{L}$. Using (12), for $X, Y \in T_{p} M$, the Ricci tensor $\operatorname{Ric}^{M}$ of $\nabla^{M}$ of $M$ is

$$
\begin{aligned}
\operatorname{Ric}^{M}(X, Y)= & \operatorname{Ric}^{L}(X, Y)-R^{L}(C, Y, C, X)-\{g(\sigma(V, Y), \sigma(X, V)) \\
& -g(\sigma(X, Y), \sigma(V, V))+\sum_{i=1}^{n}\left[g\left(\sigma\left(X_{i}, Y\right), \sigma\left(X, X_{i}\right)\right)\right. \\
& -g\left(\sigma(X, Y), \sigma\left(X_{i}, X_{i}\right)\right)+g\left(\sigma\left(\phi X_{i}, Y\right), \sigma\left(X, \phi X_{i}\right)\right) \\
& \left.\left.-g\left(\sigma(X, Y), \sigma\left(\phi X_{i}, \phi X_{i}\right)\right)\right]\right\} .
\end{aligned}
$$

Since the local Kaehler metrics $g^{\prime}$ have the same constant nonnegative holomorphic sectional curvature $\rho$, the curvature tensor of $g^{\prime}$ is given by

$$
\begin{aligned}
R^{\prime}(X, Y, Z, W)= & \frac{\rho}{4}\left\{g^{\prime}(X, Z) g^{\prime}(Y, W)-g^{\prime}(X, W) g^{\prime}(Y, Z)\right. \\
& +g^{\prime}(X, J Z) g^{\prime}(Y, J W)-g^{\prime}(X, J W) g^{\prime}(Y, J Z) \\
& \left.+2 g^{\prime}(X, J Y) g^{\prime}(Z, J W)\right\} \quad(\mathrm{cf} .[12]) .
\end{aligned}
$$

We put $\quad{ }^{W} R(X, Y, Z, W)=g\left({ }^{W} R(Z, W) Y, X\right)$. Since $\quad R^{\prime}(X, Y, Z, W)=$ $e^{-r W} R(X, Y, Z, W)$, we get

$$
\begin{aligned}
{ }^{W} R(X, Y, Z, W)= & \frac{\rho}{4} e^{-r}\{g(X, Z) g(Y, W)-g(X, W) g(Y, Z) \\
& +g(X, J Z) g(Y, J W)-g(X, J W) g(Y, J Z) \\
& +2 g(X, J Y) g(Z, J W)\}
\end{aligned}
$$


From this equation, we get

$$
{ }^{W_{R i c}}(X, Y)=\frac{\rho}{4} e^{-r}(2 n+4) g(X, Y) .
$$

From (10), for any vector fields $X, Y$ tangent to $M$, we have

$$
\begin{aligned}
R^{L}(C, Y, C, X)= & \frac{\rho}{4} e^{-r}(g(X, Y)+3 \eta(X) \eta(Y))-\frac{1}{2} g\left(\left(\left(\nabla_{X} \omega\right) Y\right) C, C\right) \\
& -\frac{1}{2} g(X, Y) g\left(\nabla_{C} B, C\right)
\end{aligned}
$$

and

$$
\operatorname{Ric}^{L}(X, Y)=\frac{\rho}{4} e^{-r}(2 n+4) g(X, Y)-n\left(\nabla_{X} \omega\right) Y-\left(\frac{1}{2} \operatorname{div} B-\frac{1}{2} n|\omega|^{2}\right) g(X, Y) .
$$

From (11) and (24), we have

$$
\left(\nabla_{X} \omega\right) Y=-\omega\left(\nabla_{X} Y\right)=\left(\left(\frac{1}{2}|\omega|-1\right) g(X, Y)+\alpha \eta(X) \eta(Y)\right)|\omega|
$$

and

(32) $\operatorname{div} B=g\left(\nabla_{C} B, C\right)+g\left(\nabla_{V} B, V\right)+\sum_{i=1}^{n} g\left(\nabla_{X_{i}} B, X_{i}\right)+\sum_{i=1}^{n} g\left(\nabla_{J X_{i}} B, J X_{i}\right)$

$$
=g\left(\nabla_{C} B, C\right)+(2 n+1)|\omega|\left(\frac{1}{2}|\omega|-1\right)+\alpha|\omega| .
$$

By using (29), (30), (31) and (32), we have

$$
\begin{aligned}
& \operatorname{Ric}^{L}(X, Y)-R^{L}(C, Y, C, X) \\
& =\frac{\rho}{4} e^{-r}\{(2 n+3) g(X, Y)-3 \eta(X) \eta(Y)\} \\
& \quad+\left(-\frac{n}{2}|\omega|^{2}+2 n|\omega|-\frac{1}{2} \alpha|\omega|\right) g(X, Y)-\left(n|\omega|-\frac{1}{2}|\omega|\right) \alpha \eta(X) \eta(Y) .
\end{aligned}
$$

From (24), we obtain

$$
\begin{aligned}
& g(\sigma(V, Y), \sigma(X, V))+\sum_{i=1}^{n}\left[g\left(\sigma\left(X_{i}, X\right), \sigma\left(X_{i}, Y\right)\right)+g\left(\sigma\left(\phi X_{i}, X\right), \sigma\left(\phi X_{i}, Y\right)\right)\right] \\
& \quad=\left(\frac{1}{2}|\omega|-1\right)^{2} g(X, Y)+\left(\alpha^{2}+\alpha|\omega|-2 \alpha\right) \eta(X) \eta(Y)
\end{aligned}
$$


Thus, since $\sigma(V, V)=-((1 / 2)|\omega|-1+\alpha) C$ and $\sigma\left(X_{i}, X_{i}\right)=-((1 / 2)|\omega|-1) C$, we have

$$
\begin{aligned}
& -\left\{g(\sigma(V, Y), \sigma(X, V))-g(\sigma(X, Y), \sigma(V, V))+\sum_{i=1}^{n}\left[g\left(\sigma\left(X_{i}, Y\right), \sigma\left(X, X_{i}\right)\right)\right.\right. \\
& \left.\left.-g\left(\sigma(X, Y), \sigma\left(X_{i}, X_{i}\right)\right)+g\left(\sigma\left(\phi X_{i}, Y\right), \sigma\left(X, \phi X_{i}\right)\right)-g\left(\sigma(X, Y), \sigma\left(\phi X_{i}, \phi X_{i}\right)\right)\right]\right\} \\
= & -\left(\frac{1}{2}|\omega|-1\right)^{2} g(X, Y)-\left(\alpha^{2}+\alpha|\omega|-2 \alpha\right) \eta(X) \eta(Y) \\
& +(2 n+1)\left\{\left(\frac{1}{2}|\omega|-1\right)^{2} g(X, Y)+\left(\frac{1}{2}|\omega|-1\right) \alpha \eta(X) \eta(Y)\right\} \\
& +\alpha^{2} \eta(X) \eta(Y)+\left(\frac{1}{2}|\omega|-1\right) \alpha g(X, Y) \\
= & \left(\frac{n}{2}|\omega|^{2}-2 n|\omega|+\frac{1}{2} \alpha|\omega|+2 n-\alpha\right) g(X, Y) \\
& +\left(n|\omega|-\frac{1}{2}|\omega|-2 n+1\right) \alpha \eta(X) \eta(Y) .
\end{aligned}
$$

Using (33) and (35), from (25), we have

$$
\operatorname{Ric}^{M}(X, Y)=\beta g(X, Y)+\gamma \eta(X) \eta(Y),
$$

where $\beta=2 n-\alpha+(\rho / 4) e^{-r}(2 n+3)$ and $\gamma=\alpha(1-2 n)-(3 / 4) \rho e^{-r}$. It is known that if all local Kaehler metrics $g^{\prime}=e^{-r} g$ of $L$ have the same constant holomorphic sectional curvature $\rho$, then $\rho=0$ or $L$ is a globally conformal Kaehler manifold (cf. [11]). Since $B \in T M^{\perp}$, for tangent vector field $X$ on $M, X(r)=$ $d r(X)=\omega(X)=0$. Hence $r$ is constant on $M$. Since $M$ is a Sasakian manifold, $\beta$ and $\gamma$ are constant on $M$ and $\beta+\gamma=2 n$ (cf. [12]). Thus, $\alpha=(\rho / 4) e^{-r}=$ constant on $M$ and $\beta=2 n+(\rho / 2) e^{-r}(n+1), \gamma=-(\rho / 2) e^{-r}(n+1) \leq 0$. Therefore, from Theorem 1, $M$ admits an Einstein-Weyl structure.

EXAMPLE. Let $H_{\lambda}^{n+1}$ be a Hopf manifold. $H_{\lambda}^{n+1}$ is isometric to $S^{1} \times S^{2 n+1}$, where $S^{2 n+1}$ is the unit sphere in $C^{n+1}$ with constant curvature 1 . Let $\omega$ be the Lee form of $H_{\lambda}^{n+1}$. The sphere $S^{2 n+1}$ is a leaf of the canonical foliation given by the Lee form $\omega=0$. Since the local Kaehler metric of $H_{\lambda}^{n+1}$ is flat and $S^{2 n+1}$ admits a Sasakian structure, $S^{2 n+1}$ admits an Einstein-Weyl structure.

Next, we shall consider generalized Hopf manifolds. 
Let $(M, \phi, V, \eta, g)$ be a Sasakian manifold and $L=M \times R$. We set

$$
\begin{gathered}
J\left(X, x \frac{\partial}{\partial s}\right)=\left(\phi X+x V,-\eta(X) \frac{\partial}{\partial s}\right), \\
h\left(\left(X, x \frac{\partial}{\partial s}\right),\left(Y, y \frac{\partial}{\partial s}\right)\right)=g(X, Y)+x y,
\end{gathered}
$$

where $X, Y$ are vector fields on $M$ and $x, y$ functions on $L$. Then $(L, J, h)$ is a generalized Hopf manifold with Lee form $\omega=2 d s$ and Lee vector field $B=2(\partial /(\partial s))$ (cf. [10]). Therefore $S^{2 n+1}(k) \times R$ is a generalized Hopf manifold.

Let $(L, J, h)$ be a generalized Hopf manifold. Then $|\omega|$ is constant. We set

$$
c=\frac{|\omega|}{2}, \quad u=\frac{\omega}{|\omega|}, \quad v=-u \circ J, \quad C=\frac{B}{|\omega|}, \quad \tilde{V}=J C .
$$

Then we have $d v=c(\Omega+2 u \wedge v)$.

Let $\mathscr{M}$ be the canonical foliation of a generalized Hopf manifold $(L, J, h)$ and $M$ be a leaf of $\mathscr{M}$. Then $M$ is a totally geodesic submanifold of $L$. We set

$$
V=\frac{1}{c} \tilde{V}_{\mid M}, \quad \eta=c v, \quad g=c^{2} h_{\mid M}, \quad \phi=J+\eta \otimes C_{\mid M} .
$$

Then $(M, \phi, V, \eta, g)$ admits a Sasakian structure (cf. [10]).

From a Theorem of Vaisman [10] and Theorem 2, we have following.

THEOREM 4. Let $L$ be a complete and simply connected generalized Hopf manifold and every leaf $M$ of the canonical foliation $\mathscr{M}$ be of constant $\phi$-sectional curvature $k$. If $k \geq 1$, then $M$ admits an Einstein-Weyl structure.

REMARK 3. In a generalized Hopf manifold $(L, h)$, if all the local Kaehler metrics $g^{\prime}=e^{-r} h$ have the same constant holomorphic sectional curvature $\rho$, then every leaf $M$ of the canonical foliation is of constant $\phi$-sectional curvature $k=\left(1 / c^{2}\right) \rho e^{-r}+1$, where $c=(|\omega| / 2)$. But converse is not true.

ACKNOWLEDGEMENT. The author would like to thank the referee for his kind advice and useful comments.

\section{References}

[ 1 ] D. E. Blair, Contact manifolds in Riemannian geometry, Lecture Notes in Math. 509, Springer, Berlin, (1976).

[2] T. Higa, Weyl manifolds and Einstein-Weyl manifolds, Comm. Math. Univ. Saint Pauli, 42 (1993) $143-160$. 
[3] J. C. Marrero and J. Rocha, Locally conformal Kaehler submersion, Geom. Dedicata, 52 (1994), 271-286.

[4] F. Narita, CR-submanifolds of locally conformal Kaehler manifolds and Riemannian submersions, Colloq. Math., 70 (1996), 165-179.

[5] F. Narita, Riemannian submersions and Riemannian manifolds with Einstein-Weyl structures, Geom. Dedicata, 65 (1997), 103-116.

[ 6] B. O'Neill, The fundamental equations of a submersion, Michigan Math. J., 13 (1966), 1-20.

[7] H. Pedersen and A. Swann, Riemannian submersions, four-manifolds and Einstein-Weyl geometry, Proc. London Math., 66 (1993), 381-399.

[ 8 ] S. Tanno, Sasakian manifolds with constant $\phi$-holomorphic sectional curvature Töhoku Math. J., 21 (1969), 501-507.

[ 9 ] I. Vaisman, On locally conformal almost Kähler manifolds, Israel J. Math., 24 (1976), 338-351.

[10] I. Vaisman, Locally conformal Kähler manifolds with parallel Lee form, Rend. Mat. Roma, 12 (1979), 263-284.

[11] I. Vaisman, Some curvature properties of locally conformal Kaehler manifolds, Trans. Amer. Math. Soc., (2) 259 (1980), 439-447.

[12] K. Yano and M. Kon, Structures on manifolds, World Sci. Singapore, (1984).

Department of Mathematics

Akita National College of Technology

Akita 011-8511 Japan

E-mail: narifumi@ipc.akita-nct.ac.jp 